\title{
Combination of isoliquiritigenin and tumor necrosis factor-related apoptosis-inducing ligand induces apoptosis in colon cancer HT29 cells
}

\author{
Tatsushi Yoshida · Mano Horinaka · Mami Takara • \\ Mayuko Tsuchihashi · Nobuhiro Mukai • \\ Miki Wakada · Toshiyuki Sakai
}

Received: 28 March 2008/Accepted: 2 June 2008/Published online: 31 July 2008

(C) The Japanese Society for Hygiene 2008

\begin{abstract}
Objectives Isoliquiritigenin is a chalcone derivative with potential in cancer chemoprevention. Although tumor necrosis factor-related apoptosis-inducing ligand (TRAIL) is a promising anti-cancer agent, some cancer cells are resistant to TRAIL treatment. Current studies have tried to overcome TRAIL-resistant cancer cells. Here, we show for the first time that isoliquiritigenin overcomes TRAIL resistance in colon cancer HT29 cells.

Methods HT29 cells were treated with isoliquiritigenin and/or TRAIL, and apoptosis induction was detected by flow cytometry and fluorescence microscopy. Protein expression relating to the TRAIL pathway was analyzed by Western blotting.

Results A single treatment with isoliquiritigenin scarcely induced apoptosis in HT29 cells. Combined treatment with suboptimal concentrations of isoliquiritigenin and TRAIL markedly induced apoptosis, however. The effect was blocked by a pan-caspase inhibitor and a caspase-3, 8, 9, or 10 inhibitor, suggesting that the combination facilitates caspase-dependent apoptosis. Furthermore, the apoptosis induced by isoliquiritigenin and TRAIL was blocked by a dominant negative form of the TRAIL receptor. This result indicates that the combined effect is caused by specific interaction between TRAIL and its receptors. Isoliquiritigenin increased the amount of DR5 protein among TRAIL receptors. Isoliquiritigenin did not significantly increase levels of the Bcl-2 family proteins Bcl-2, Bcl-xL, and BAX.
\end{abstract}

T. Yoshida $\cdot$ M. Horinaka $\cdot$ M. Takara $\cdot$ M. Tsuchihashi ·

N. Mukai $\cdot$ M. Wakada $\cdot$ T. Sakai $(\bowtie)$

Department of Molecular-Targeting Cancer Prevention,

Graduate School of Medical Science, Kyoto Prefectural

University of Medicine, Kawaramachi-Hirokoji,

Kamigyo-ku, Kyoto 602-8566, Japan

e-mail: tsakai@koto.kpu-m.ac.jp
Conclusions Our results suggest that isoliquiritigenin has the potential to overcome resistance to TRAIL in cancer cells and its chemopreventive effects may depend on TRAIL function.

Keywords Isoliquiritigenin - Chalcone - DR5 · TRAIL · Apoptosis
Abbreviations
DR5 Death receptor 5
TRAIL Tumor necrosis factor-related apoptosis-inducing ligand
TNF Tumor necrosis factor
Bcl-2 B cell lymphoma-2
BAX Bcl-2-associated $\mathrm{X}$ protein

\section{Introduction}

The risk of human death is highest from malignant tumors. Thus, prevention of malignant tumors is the most important issue in the field of preventive medicine. Many epidemiological studies have attempted to reveal a relationship between foods and carcinogenesis. Foods have been considered to be related to the prevention of carcinogenesis. In addition, there is much evidence that dietary components are able to prevent carcinogenesis in animal models [1-3]. Isoliquiritigenin is a chalcone derivative contained in edible plants and used as a Chinese herbal medicine. Isoliquiritigenin has potential as a chemopreventive agent and has exhibited anti-carcinogenic effects in several experimental models. Isoliquiritigenin inhibits aberrant crypt foci from developing [4, 5], skin tumors [6], and pulmonary metastasis of renal cell carcinoma [7] in mice. 
Moreover, studies in vitro show that isoliquiritigenin directly induces apoptosis in different types of cancer cell, such as prostate [8], lung [9], and gastric [10] cancer cells, hepatoma cells [11], and melanoma cells [12]. In addition, isoliquiritigenin suppresses ErbB3 signaling and the PI3K/ Akt pathways [13] and the NF-kB pathway [14, 15], which relate to cancer progression. Thus, isoliquiritigenin is an attractive agent in cancer prevention and therapy.

Tumor necrosis factor (TNF)-related apoptosis-inducing ligand (TRAIL), a member of the TNF superfamily, has been identified as an expressed sequenced tag (EST) showing homology with TNF [16]. TRAIL kills many types of cancer cell in vitro and in vivo, with little or no toxic effect on normal cells $[17,18]$. The property is very useful for an anti-cancer agent. Not only recombinant human TRAIL but also agonistic antibodies for TRAIL receptors have been developed as anti-cancer agents [19, 20]. TRAIL or agonistic TRAIL-receptor antibodies are undergoing Phase I/II clinical trials in patients with solid malignant tumors or non-Hodgkin lymphomas [21]. To date, five TRAIL receptors have been reported: death receptor (DR) 5, also called TRAIL-R2, TRICK2 or KILLER [22-26], DR4, decoy receptor (DcR) 1, DcR2, and osteoprotegerin [27]. Only DR4 and DR5 can mediate TRAIL-induced apoptosis. The other receptors play a dominant negative role by competing with DR4 and DR5 for interaction with TRAIL. DR4 and DR5 bind to procaspase- 8 and 10 through the adapter protein FAS-associated death domain (FADD), resulting in formation of a death-inducing signaling complex (DISC) [27]. Caspase-8 and 10 are auto-activated after the DISC forms. There are two different cell types based on the TRAIL-signaling pathway for apoptosis. In type I cells, activated caspase- 8 and 10 directly cleave and activate effector caspases such as caspase-3. Consequently, effector caspases cleave many substrates and cause apoptosis. On the other hand, in type II cells, the apoptotic signal is transmitted to mitochondria and the activation of caspase-9 downstream of the mitochondria leads to the cleavage and activation of effector caspases. Some malignant tumors still remain resistant to TRAIL [28]. Thus, it is important to overcome the resistance in TRAIL-based cancer treatment.

In this study we show that isoliquiritigenin increases production of DR5 protein and overcomes resistance to TRAIL in colon cancer HT29 cells.

\section{Materials and methods}

\section{Reagents}

Isoliquiritigenin was purchased from Extrasynthese (Genay, France) and dissolved in DMSO. Soluble recombinant human TRAIL/Apo2L was obtained from PeproTech (London, UK). R\&D Systems (Minneapolis, MN, USA) supplied human recombinant DR5 (TRAIL$\mathrm{R} 2) / \mathrm{Fc}$ chimeric protein and the following caspase inhibitors: pan-caspase inhibitor, zVAD-fmk; caspase-3 inhibitor, zDEVD-fmk; caspase-8 inhibitor, zIETD-fmk; caspase-9 inhibitor, zLEHD-fmk; and caspase-10 inhibitor, zAEVD-fmk.

\section{Cell culture}

Human colon cancer HT29 cells were maintained in Dulbecco's-modified Eagle medium (DMEM) supplemented with $10 \%$ fetal bovine serum, $4 \mathrm{mM}$ glutamine, $100 \mathrm{U} / \mathrm{ml}$ penicillin, and $100 \mu \mathrm{g} / \mathrm{ml}$ streptomycin. Cells were incubated at $37^{\circ} \mathrm{C}$ in a humidified atmosphere of $5 \%$ $\mathrm{CO}_{2}$.

\section{Detection of apoptosis}

For detection of the Sub-G1 population, cells were harvested from culture dishes, washed with PBS and suspended in PBS containing 0.1\% Triton-X100 and RNase A (Sigma, St Louis, MO, USA). The nuclei were stained with propidium iodide (PI). The DNA content was measured using FACS Calibur (Becton Dickinson, Franklin Lakes, NJ, USA). For each experiment, 10,000 events were collected. The data (means \pm S.D.; $n=3$ ) were analyzed with Cell Quest software (Becton Dickinson). Student's $t$ test was used.

\section{DAPI staining}

HT29 cells were plated out on six-well plates and treated with isoliquiritigenin or solvent (DMSO) for $24 \mathrm{~h}$. The cells were washed with PBS and treated with methanol for 1 min. After drying, they were treated with PBS containing 4',6-diamino-2-phenylindole (DAPI) and nuclei were observed by fluorescence microscopy.

\section{Western blot analysis}

Western blotting was performed as described previously [29]. Rabbit polyclonal anti-DR5 and DR4 (Prosci, Poway, CA, USA), DcR2 (Imgenex, San Diego, CA, USA), BAX and Bcl-xL (Santa Cruz, Santa Cruz, CA, USA) antibodies, and mouse monoclonal anti-Bcl-2 (Santa Cruz) and $\beta$-actin (Sigma) antibodies were used as the primary antibodies. The signal was detected with an ECL Western blot analysis system (GEhealthcare, Piscataway, NJ, USA). 


\section{Results}

Combined treatment with isoliquiritigenin and TRAIL induces apoptosis in colon cancer HT29 cells

TRAIL is a promising agent for cancer treatment; however, some cancer cells are resistant to it. To overcome this resistance, we searched for agents that can enhance TRAIL efficacy and identified isoliquiritigenin as a candidate. As shown in Fig. 1, colon cancer HT29 cells were resistant to TRAIL, with less than $10 \%$ of the cells undergoing apoptosis. Isoliquiritigenin is known to induce apoptosis in many types of cancer cell; it also only slightly induced apoptosis in HT29 cells, however. Next, we simultaneously used isoliquiritigenin and TRAIL. Interestingly, the combined treatment significantly induced apoptosis in HT29 cells compared with TRAIL treatment alone. Notably, $40 \mu \mathrm{M}$ isoliquiritigenin had the strongest effect with TRAIL.

Caspase inhibitors and DR5/Fc chimeric protein block the apoptosis induced by the combination of isoliquiritigenin and TRAIL

Apoptosis proceeds through the sequential cleavage and activation of caspases. To verify that the sub-G1 populations detected in Fig. 1 reflect caspase-dependent apoptosis, we used caspase inhibitors. As shown in Fig. 2a, the pan-caspase inhibitor zVAD-fmk significantly blocked apoptosis induced by the combination of isoliquiritigenin and TRAIL. Moreover, specific caspase inhibitors against caspase-3, 8, 9, and 10 also significantly inhibited the apoptosis. Taken together, these results indicate that the combination of isoliquiritigenin and TRAIL induces

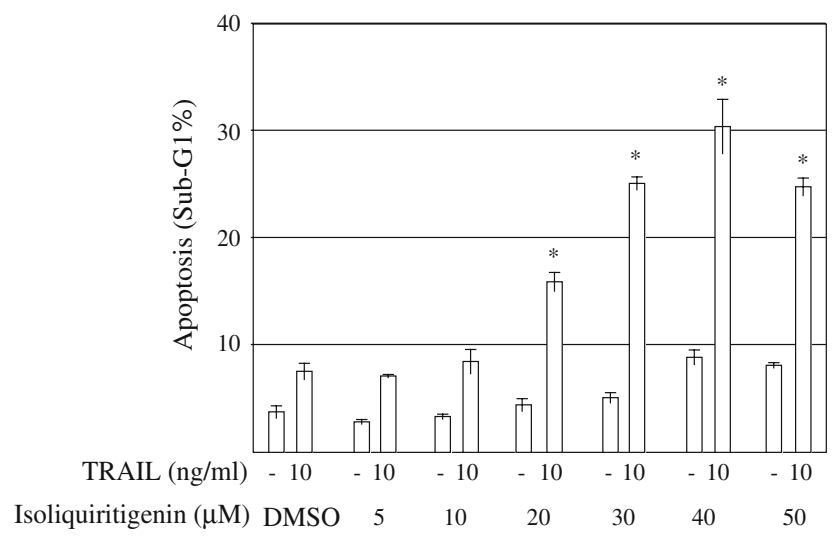

Fig. 1 Combined treatment with isoliquiritigenin and TRAIL induces apoptosis in colon cancer HT29 cells. Colon cancer HT29 cells were treated with $10 \mathrm{ng} / \mathrm{ml} \mathrm{TRAIL}$ and/or the indicated concentration of isoliquiritigenin for $24 \mathrm{~h}$. The Sub-G1 population was analyzed by flow cytometry. The values shown are means $(n=3)$; bars, \pm S.D. $* P<0.01$ when compared with the sample treated with TRAIL alone caspase-dependent apoptosis. Of note, caspase-9 inhibitor clearly abrogated the apoptosis induction. Caspase-9 plays a role in apoptosis downstream mitochondria and mediates the apoptotic signal to effector caspase- 3 in type II cells. On the other hand, in type I cells, TRAIL induces apoptosis without a mitochondrial pathway. Therefore, these results indicate that the combination of isoliquiritigenin and TRAIL induces caspase-dependent apoptosis through a
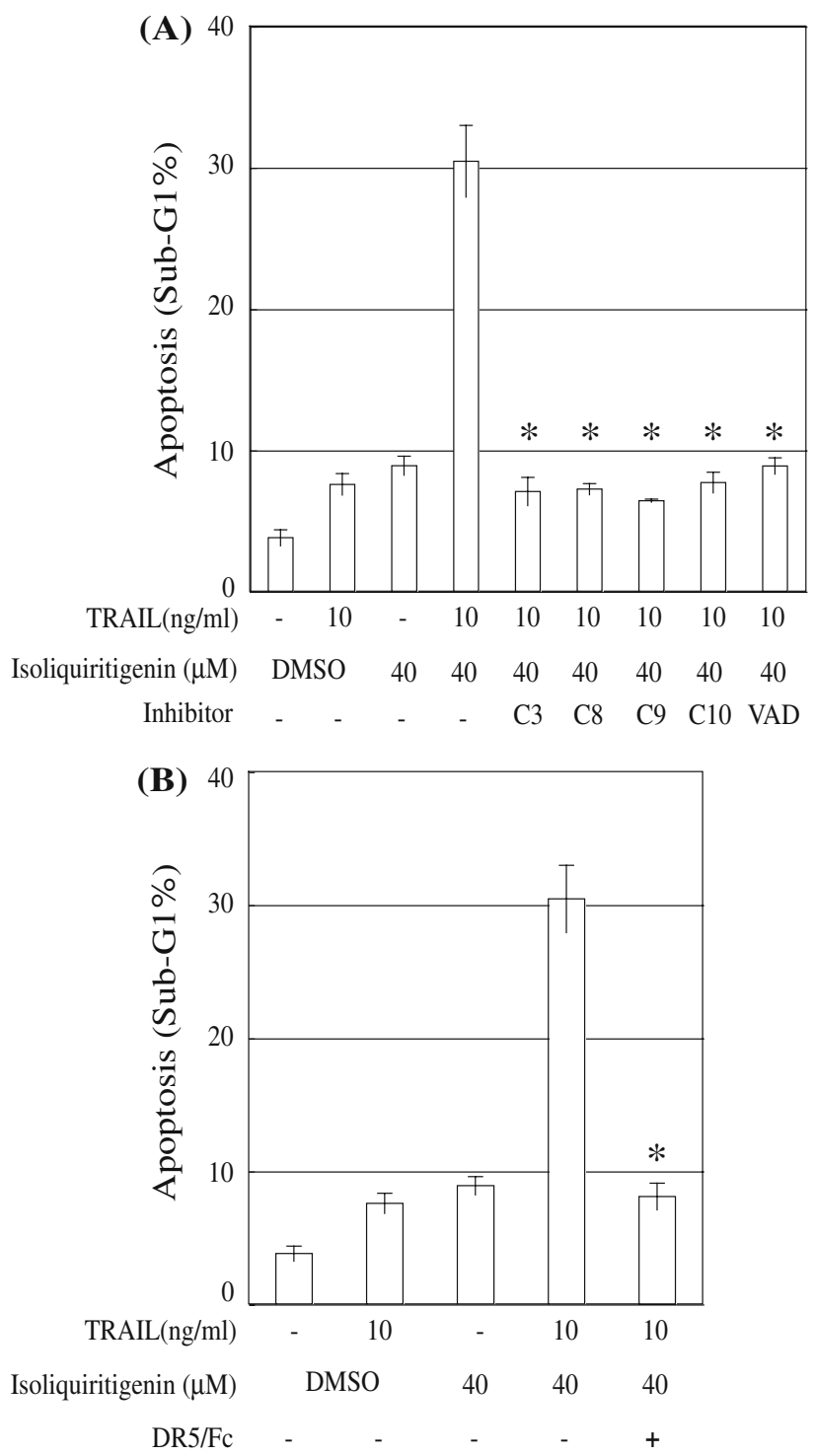

Fig. 2 Caspase inhibitors and DR5/Fc chimeric protein block apoptosis induced by the combination of isoliquiritigenin and TRAIL. Colon cancer HT29 cells were treated with $10 \mathrm{ng} / \mathrm{ml}$ TRAIL and/or $40 \mu \mathrm{M}$ isoliquiritigenin with or without caspase inhibitors (a) or DR5/ $\mathrm{Fc}$ chimeric protein (b) for $24 \mathrm{~h}$. The Sub-G1 population was analyzed by flow cytometry. The values shown are means $(n=3)$; bars, \pm S.D. $C 3$, caspase-3 inhibitor; $C 8$, caspase-8 inhibitor; $C 9$, caspase-9 inhibitor; $C 10$, caspase-10 inhibitor; $V A D$, pan-caspase inhibitor. $* P<0.01$ when compared with the sample treated with isoliquiritigenin and TRAIL 
mitochondrial pathway dependent on caspase-9 in a type II cell manner. TRAIL causes apoptosis by interacting with specific pro-apoptotic receptors, DR5 and DR4. To examine whether the combination of isoliquiritigenin and TRAIL induces apoptosis through TRAIL receptors, we used a DR5/Fc chimeric protein which has a dominant negative effect, because it can interact with TRAIL but not mediate apoptosis. As shown in Fig. 2b, the DR5/Fc chimera abrogated the apoptosis induced by the combined treatment. The result suggests that the combination of isoliquiritigenin and TRAIL induces apoptosis through a TRAIL-signaling pathway mediated by TRAIL receptors but not in a non-specific manner.

Combination of isoliquiritigenin and TRAIL induces nuclear fragmentation

Next, we observed nuclei treated with isoliquiritigenin and/ or TRAIL using DAPI staining (Fig. 3). In apoptotic cells, nuclei are condensed and fragmented. As a single treatment, neither isoliquiritigenin nor TRAIL had any effect. The image of nuclei was similar to that of cells treated with the solvent DMSO. Cells treated with both isoliquiritigenin and TRAIL had fragmented and condensed nuclei, suggesting that only combined treatment drastically induced apoptosis consistent with the sub-G1 data in Fig. 1. Moreover, the pan-caspase inhibitor zVAD-fmk blocked the nuclear fragmentation and condensation induced by the combination of isoliquiritigenin and TRAIL, indicating that the change of nuclear image is mediated via the activation of caspases.

Isoliquiritigenin up-regulates a TRAIL receptor DR5 protein

We hypothesized that isoliquiritigenin regulates proteins which relate to TRAIL signaling and enhances TRAILinduced apoptosis. We therefore examined whether isoliquiritigenin alters the expression of proteins which act on TRAIL-induced apoptosis. First, we examined protein levels of TRAIL receptors. Isoliquiritigenin increased the amount of pro-apoptotic receptor DR5 in a dose-dependent manner (Fig. 4). As previously shown [29], DR5 protein furnishes two bands. An upper band was barely detectable but a lower band was increased by the isoliquiritigenin treatment. Moreover, neither DR4 nor DcR2 was apparently affected by isoliquiritigenin. DcR1 protein was not detected (data not shown). Bcl-2 family proteins are key factors in the induction of apoptosis. Bcl-2 and Bcl-xL can block apoptosis induced by TRAIL [30, 31]. Conversely, BAX facilitates TRAIL-induced apoptosis, and a deficiency of BAX abrogates TRAIL-induced apoptosis [32]. We performed Western blotting of Bcl-2, Bcl-xL, and BAX. Isoliquiritigenin did not affect the levels of these Bcl-2 family proteins in a dose-dependent manner. Taken together, the results suggest that isoliquiritigenin increases the amount of DR5 protein and enhances apoptosis induced by TRAIL.
Fig. 3 The combination of isoliquiritigenin and TRAIL causes nuclear fragmentation in HT29 cells. Colon cancer HT29 cells were treated with $10 \mathrm{ng} / \mathrm{ml}$ TRAIL and/or $40 \mu \mathrm{M}$ isoliquiritigenin for $24 \mathrm{~h}$. Nuclei were stained with DAPI after methanol fixation and observed by fluorescence microscopy. The pan-caspase inhibitor zVAD-fmk was added at the same time as TRAIL and isoliquiritigenin. Apoptotic cells are indicated with arrows

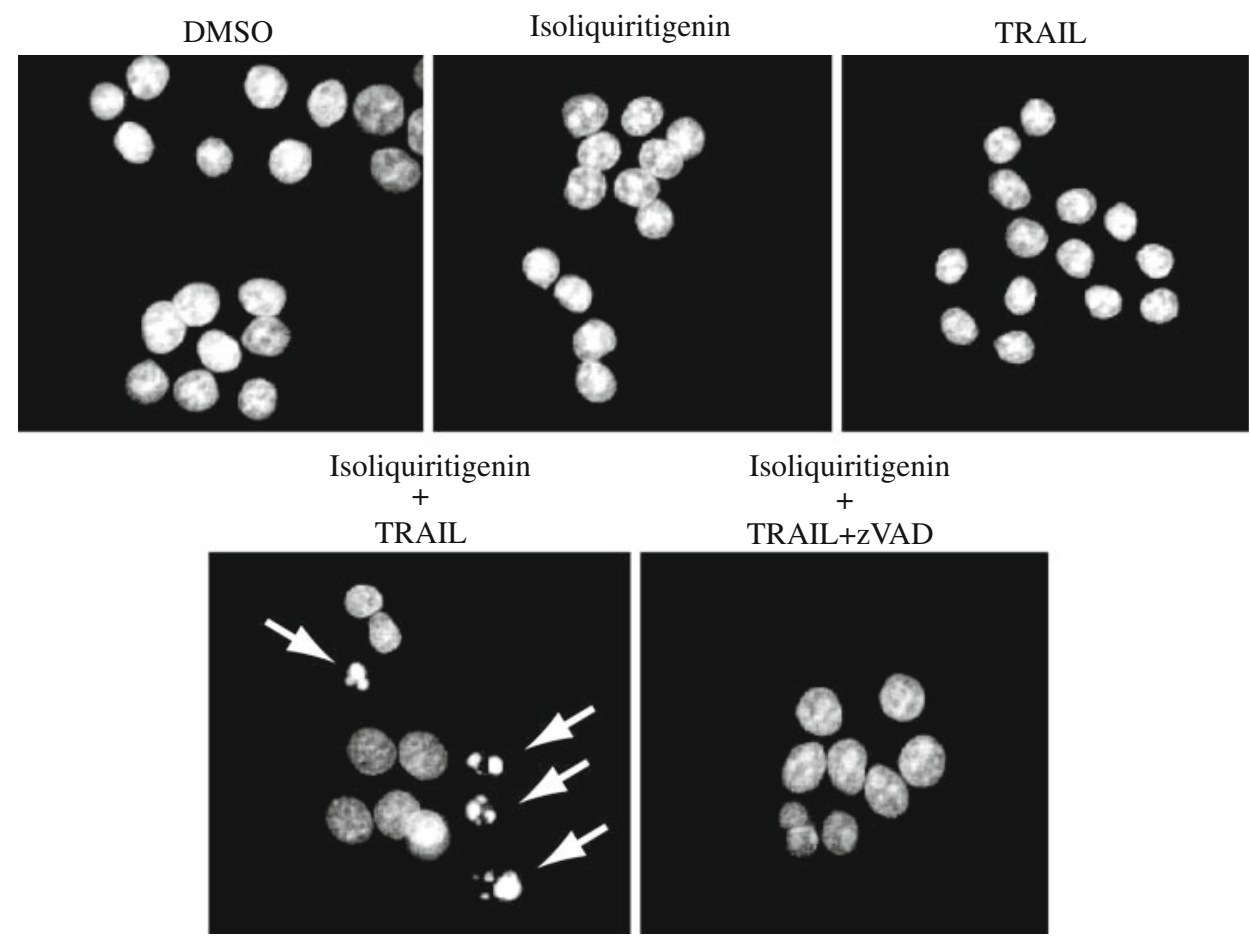


Fig. 4 The combination of isoliquiritigenin and TRAIL upregulates the TRAIL receptor DR5. a HT29 cells were treated with the indicated concentrations of isoliquiritigenin for $24 \mathrm{~h}$. Western blotting was performed with anti-DR5, DR4, and DcR2 antibodies. $\beta$-Actin was used as a loading control. As a positive control, a DR5 protein pattern after a tunicamycin treatment is shown [34]. b HT29 cells were treated as shown in $\mathbf{a}$. Western blotting was performed with anti-Bcl-2, Bcl-xL, and BAX antibodies. $\beta$-Actin was used as a loading control. $C T$, treated with solvent DMSO. Arrows, DR5 protein bands; asterisks, non-specific bands. Band intensity is analyzed by ImageJ software and shown as bar graphs
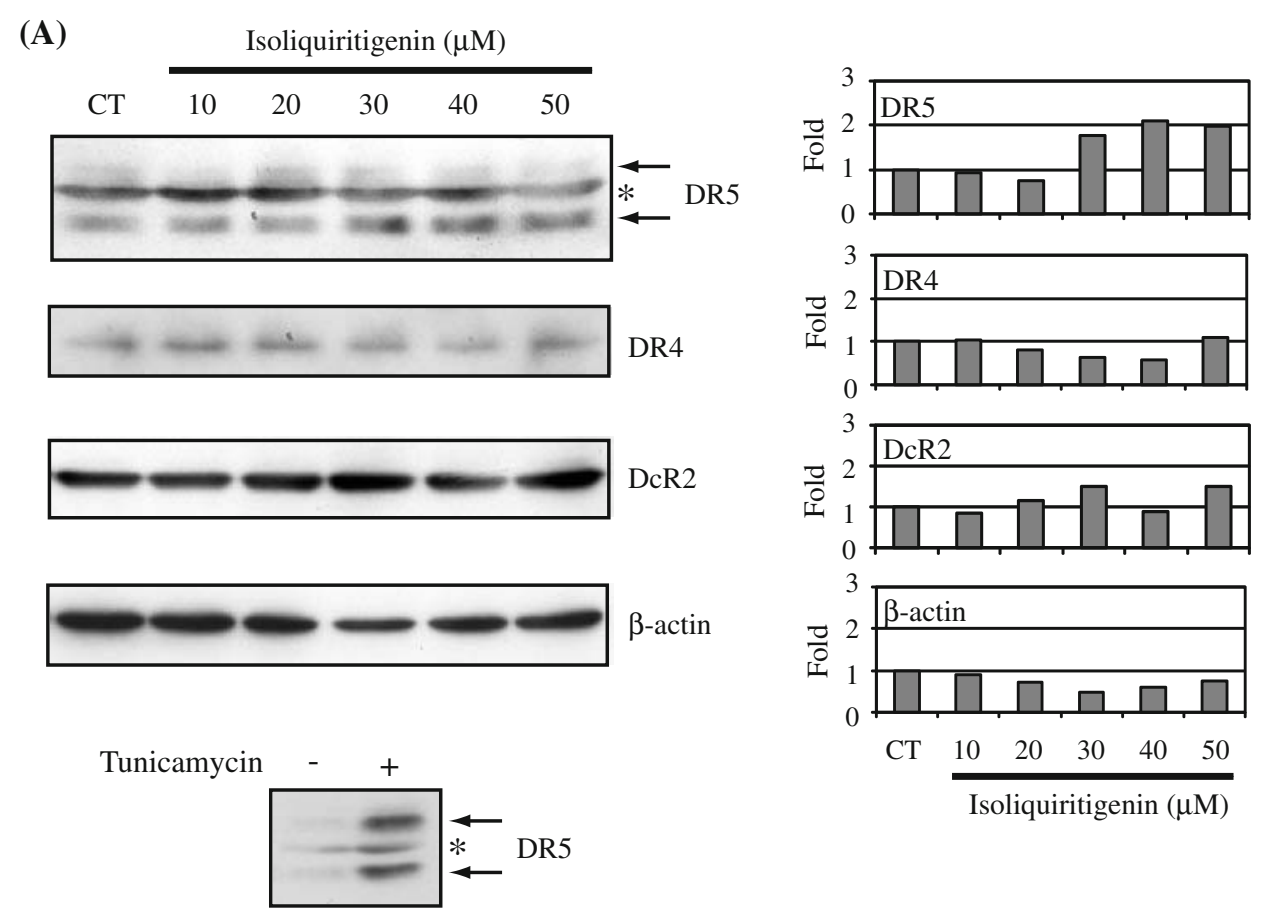

(B)
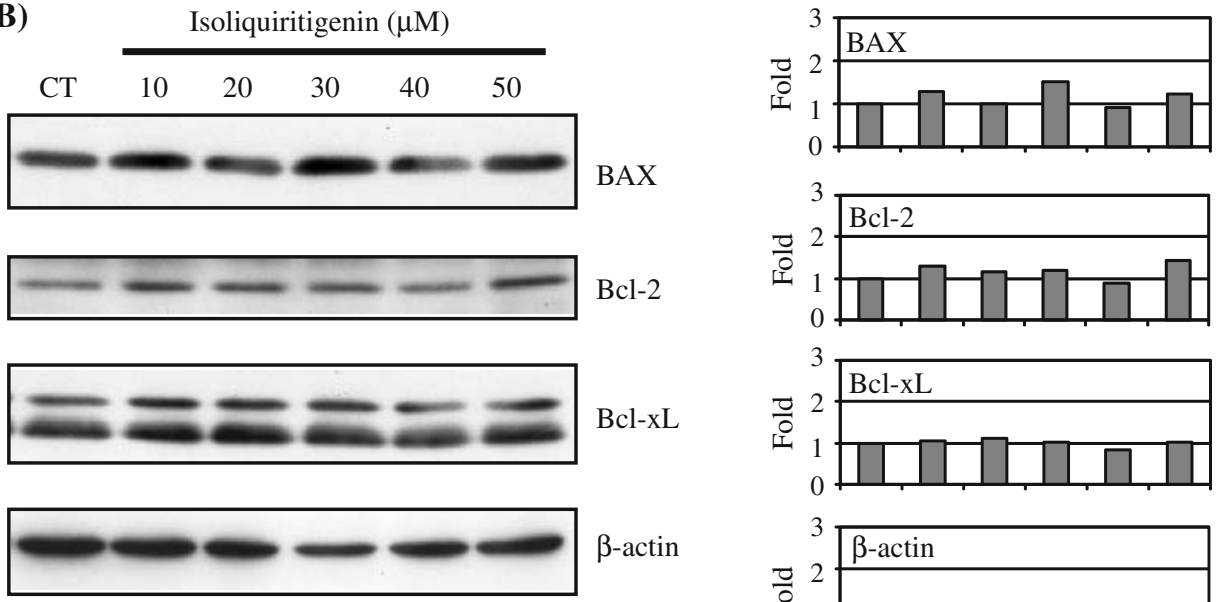

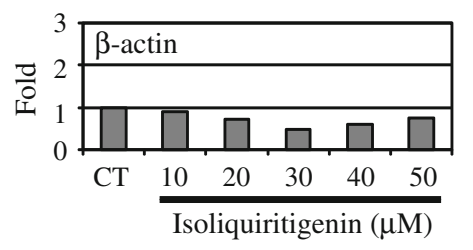

\section{Discussion}

We showed here that the combination of suboptimal concentrations of isoliquiritigenin and TRAIL drastically induces apoptosis in colon cancer HT29 cells. Interestingly, treatment with either isoliquiritigenin or TRAIL alone hardly induced any apoptosis in HT29 cells. The results mean that the combination of these agents generates an effect that is more than simply additive. As the effect of single agents is becoming limited and the identification of new agents is generally difficult, it is promising that a combination of agents can function synergistically. As a possible mechanism by which isoliquiritigenin enhances
TRAIL efficacy, we showed that isoliquiritigenin increases the level of DR5 but not DR4. These results indicate that the regulation of each TRAIL receptor is different from that of other receptors. We previously reported the sequence of a promoter region in DR5, different from that in DR4, and suggested that the regulation is independent [33]. This hypothesis is consistent with the current findings. In addition, we previously reported that TRAIL efficacy is facilitated by agents which can up-regulate DR5 expression, for example histone deacetylase inhibitors [29], tunicamycin [34], proteasome inhibitor [35], flavonoids [36-38], and carotenoids [39]. To enhance the effect of an agent which acts as a ligand against a receptor, combined 


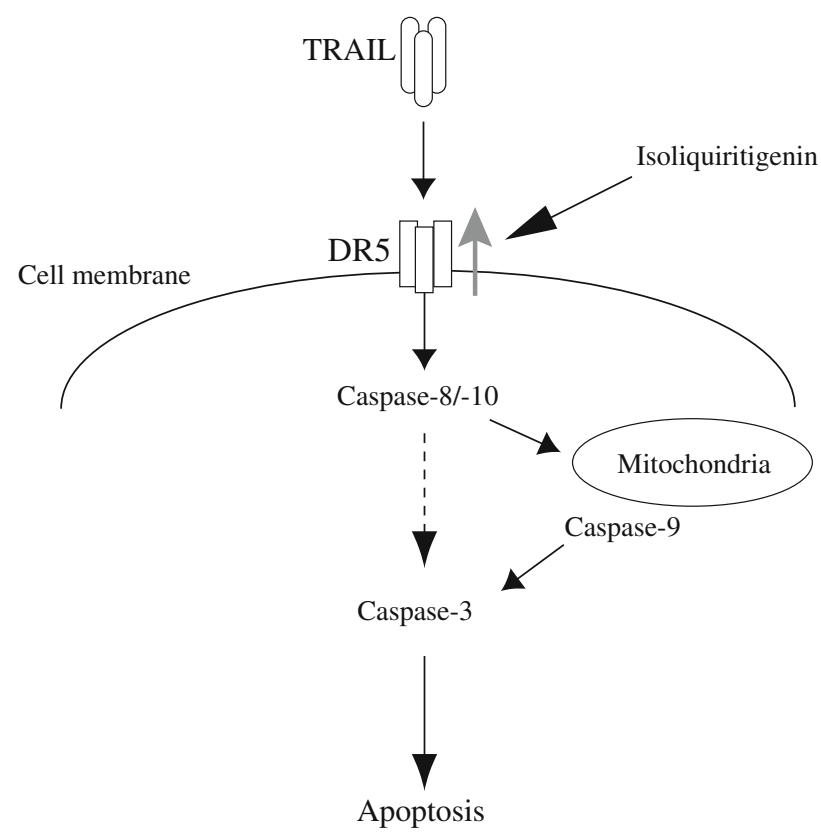

Fig. 5 Schematic model. The combination of isoliquiritigenin and TRAIL markedly induces apoptosis in HT29 cells. Isoliquiritigenin up-regulates DR5 expression, which is considered to enhance TRAIL action. Enhanced TRAIL signaling results in apoptosis through the activation of caspase-8/10, 9 and 3. Caspase-8/10 can activate caspase- 3 via caspase- 9 , which acts downstream of mitochondria

treatment with another agent which can increase the level of the receptor is a very promising strategy.

The tumor-suppressor p53 is important in cancer prevention and more than half of malignant tumors have p53 mutations [40]. p53 contributes to sensitivity of cancer cells to anti-cancer agents [41] and DR5 is downstream of p53 [26, 42]. However, HT29 cells have a p53 mutation, indicating that DR5 up-regulation by isoliquiritigenin is independent of p53. In addition, the combination of isoliquiritigenin and TRAIL can induce apoptosis independent of p53 status.

Isoliquiritigenin has had chemopreventive effects against malignant tumors in animal studies [4-7]; however the mechanism underlying these effects has not been elucidated completely. TRAIL, an endogenous protein expressed in many types of cell, causes an anti-tumor immune response [43]. However, a recombinant human TRAIL protein was used in the current study. Therefore, isoliquiritigenin may exert a chemopreventive effect in cooperation with endogenous TRAIL in vivo. Recently, it has been reported that a deficiency of DR5 promoted tumorigenesis and enhanced metastasis in mice [44, 45]. The up-regulation of DR5 expression may be important to the chemopreventive effect of isoliquiritigenin. Moreover, interferon [46], retinoic acids [47, 48] and Bacillus Calmette-Guerin (BCG) [49] promote endogenous expression of TRAIL. A combination of isoliquiritigenin and these TRAIL inducers may effectively kill cancer cells.

In conclusion, isoliquiritigenin up-regulates DR5 expression, and in combination with TRAIL, markedly induces apoptosis in TRAIL-resistant colon cancer HT29 cells through activation of caspase-8, 10, 9, and 3 (Fig. 5). Our data suggest that isoliquiritigenin is useful for TRAILbased cancer treatment and that the chemopreventive effect of isoliquiritigenin may depend on TRAIL function.

Acknowledgments This work was supported in part by the Japanese Ministry of Education, Culture, Sports, Science and Technology.

\section{References}

1. Yang CS, Landau JM, Huang MT, Newmark HL. Inhibition of carcinogenesis by dietary polyphenolic compounds. Annu Rev Nutr. 2001;21:381-406.

2. Seeram NP. Berry fruits: compositional elements, biochemical activities, and the impact of their intake on human health, performance, and disease. J Agric Food Chem. 2008;56:627-9.

3. Khan N, Afaq F, Mukhtar H. Apoptosis by dietary factors: the suicide solution for delaying cancer growth. Carcinogenesis. 2007;28:233-9.

4. Baba M, Asano R, Takigami I, Takahashi T, Ohmura M, Okada $\mathrm{Y}$, et al. Studies on cancer chemoprevention by traditional folk medicines XXV. Inhibitory effect of isoliquiritigenin on azoxymethane-induced murine colon aberrant crypt focus formation and carcinogenesis. Biol Pharm Bull. 2002;25:247-50.

5. Takahashi T, Takasuka N, Iigo M, Baba M, Nishino H, Tsuda H, et al. Isoliquiritigenin, a flavonoid from licorice, reduces prostaglandin $\mathrm{E}_{2}$ and nitric oxide, causes apoptosis, and suppresses aberrant crypt foci development. Cancer Sci. 2004;95:448-53.

6. Yamamoto S, Aizu E, Jiang H, Nakadate T, Kiyoto I, Wang JC, et al. The potent anti-tumor-promoting agent isoliquiritigenin. Carcinogenesis. 1991;12:317-23.

7. Yamazaki S, Morita T, Endo H, Hamamoto T, Baba M, Joichi Y, et al. Isoliquiritigenin suppresses pulmonary metastasis of mouse renal cell carcinoma. Cancer Lett. 2002;183:23-30.

8. Jung JI, Lim SS, Choi HJ, Cho HJ, Shin HK, Kim EJ, et al. Isoliquiritigenin induces apoptosis by depolarizing mitochondrial membranes in prostate cancer cells. J Nutr Biochem. 2006;17:689-96.

9. Hsu YL, Kuo PL, Chiang LC, Lin CC. Isoliquiritigenin inhibits the proliferation and induces the apoptosis of human non-small cell lung cancer A549 cells. Clin Exp Pharmacol Physiol. 2004;31:414-8.

10. Ma J, Fu NY, Pang DB, Wu WY, Xu AL. Apoptosis induced by isoliquiritigenin in human gastric cancer MGC-803 cells. Planta Med. 2001;67:754-7.

11. Hsu YL, Kuo PL, Lin CC. Isoliquiritigenin induces apoptosis and cell cycle arrest through p53-dependent pathway in Hep G2 cells. Life Sci. 2005;77:279-92.

12. Iwashita K, Kobori M, Yamaki K, Tsushida T. Flavonoids inhibit cell growth and induce apoptosis in B16 melanoma 4A5 cells. Biosci Biotechnol Biochem. 2000;64:1813-20.

13. Jung JI, Chung E, Seon MR, Shin HK, Kim EJ, Lim SS, et al. Isoliquiritigenin (ISL) inhibits ErbB3 signaling in prostate cancer cells. Biofactors. 2006;28:159-68.

14. Kumar S, Sharma A, Madan B, Singhal V, Ghosh B. Isoliquiritigenin inhibits IkappaB kinase activity and ROS generation to block TNF-alpha induced expression of cell adhesion molecules 
on human endothelial cells. Biochem Pharmacol. 2007;73:160212 .

15. Kwon HM, Choi YJ, Choi JS, Kang SW, Bae JY, Kang IJ, et al. Blockade of cytokine-induced endothelial cell adhesion molecule expression by licorice isoliquiritigenin through NF-kappaB signal disruption. Exp Biol Med (Maywood). 2007;232:235-45.

16. Pitti RM, Marsters SA, Ruppert S, Donahue CJ, Moore A, Ashkenazi A. Induction of apoptosis by Apo-2 ligand, a new member of the tumor necrosis factor cytokine family. J Biol Chem. 1996;271:12687-90.

17. Walczak H, Miller RE, Ariail K, Gliniak B, Griffith TS, Kubin M, et al. Tumoricidal activity of tumor necrosis factor-related apoptosis-inducing ligand in vivo. Nat Med. 1999;5:157-63.

18. Ashkenazi A, Pai RC, Fong S, Leung S, Lawrence DA, Marsters SA, et al. Safety and antitumor activity of recombinant soluble Apo2 ligand. J Clin Invest. 1999;104:155-62.

19. Ichikawa K, Liu W, Zhao L, Wang Z, Liu D, Ohtsuka T, et al. Tumoricidal activity of a novel anti-human DR5 monoclonal antibody without hepatocyte cytotoxicity. Nat Med. 2001;7:95460.

20. Pukac L, Kanakaraj P, Humphreys R, Alderson R, Bloom M, Sung C, et al. HGS-ETR1, a fully human TRAIL-receptor 1 monoclonal antibody, induces cell death in multiple tumour types in vitro and in vivo. Br J Cancer. 2005;92:1430-41.

21. Fesik SW. Promoting apoptosis as a strategy for cancer drug discovery. Nat Rev Cancer. 2005;5:876-85.

22. Pan G, Ni J, Wei Y-F, Yu G-L, Gentz R, Dixit VM. An antagonist decoy receptor and a death domain-containing receptor for TRAIL. Science. 1997;277:815-8.

23. Sheridan JP, Marsters SA, Pitti RM, Gurney A, Skubatch M, Baldwin D, et al. Control of TRAIL-induced apoptosis by a family of signaling and decoy receptors. Science. 1997;277:81821.

24. Screaton GR, Mongkolsapaya J, Xu X-N, Cowper AE, McMichael AJ, Bell JI. TRICK2, a new alternatively spliced receptor that transduces the cytotoxic signal from TRAIL. Curr Biol. 1997; 7:693-6.

25. Walczak H, Degli-Esposti MA, Johnson RS, Smolak PJ, Waugh JY, Boiani N, et al. TRAIL-R2: a novel apoptosis-mediating receptor for TRAIL. EMBO J. 1997;16:5386-97.

26. Wu GS, Burns TF, McDonald ER 3rd, Jiang W, Meng R, Krantz ID, et al. KILLER/DR5 is a DNA damage-inducible p53-regulated death receptor gene. Nat Genet. 1997;17:141-3.

27. LeBlanc HN, Ashkenazi A. Apo2L/TRAIL and its death and decoy receptors. Cell Death Differ. 2003;10:66-75.

28. Zhang L, Fang B. Mechanisms of resistance to TRAIL-induced apoptosis in cancer. Cancer Gene Ther. 2005;12:228-37.

29. Nakata S, Yoshida T, Horinaka M, Shiraishi T, Wakada M, Sakai T. Histone deacetylase inhibitors up-regulate death receptor 5/ TRAIL-R2, and sensitize apoptosis induced by TRAIL/APO2-L in human malignant tumor cells. Oncogene. 2004;23:6261-71.

30. Fulda S, Meyer E, Debatin KM. Inhibition of TRAIL-induced apoptosis by Bcl-2 overexpression. Oncogene. 2002;21:2283-94.

31. Hetschko H, Voss V, Horn S, Seifert V, Prehn JH, Kögel D. Pharmacological inhibition of Bcl-2 family members reactivates TRAIL-induced apoptosis in malignant glioma. J Neurooncol. 2008;86:265-72.

32. LeBlanc H, Lawrence D, Varfolomeev E, Totpal K, Morlan J, Schow $\mathrm{P}$, et al. Tumor-cell resistance to death receptor-induced apoptosis through mutational inactivation of the proapoptotic Bcl-2 homolog Bax. Nat Med. 2002;8:274-81.
33. Yoshida T, Sakai T. Promoter of TRAIL-R2 gene. Vitam Horm. 2004;67:35-49.

34. Shiraishi T, Yoshida T, Nakata S, Horinaka M, Wakada M, Mizutani Y, et al. Tunicamycin enhances tumor necrosis factorrelated apoptosis-inducing ligand-induced apoptosis in human prostate cancer cells. Cancer Res. 2005;65:6364-70.

35. Yoshida T, Shiraishi T, Nakata S, Horinaka M, Wakada M, Mizutani Y, et al. Proteasome inhibitor MG132 induces death receptor 5 through CCAAT/enhancer-binding protein homologous protein. Cancer Res. 2005;65:5662-7.

36. Horinaka M, Yoshida T, Shiraishi T, Nakata S, Wakada M, Nakanishi R, et al. Luteolin induces apoptosis via death receptor 5 upregulation in human malignant tumor cells. Oncogene. 2005;24:7180-9.

37. Horinaka M, Yoshida T, Shiraishi T, Nakata S, Wakada M, Nakanishi $R$, et al. The combination of TRAIL and luteolin enhances apoptosis in human cervical cancer HeLa cells. Biochem Biophys Res Commun. 2005;333:833-8.

38. Horinaka M, Yoshida T, Shiraishi T, Nakata S, Wakada M, Sakai $\mathrm{T}$. The dietary flavonoid apigenin sensitizes malignant tumor cells to tumor necrosis factor-related apoptosis-inducing ligand. Mol Cancer Ther. 2006;5:945-51.

39. Yoshida T, Maoka T, Das SK, Kanazawa K, Horinaka M, Wakada M, et al. Halocynthiaxanthin and peridinin sensitize colon cancer cell lines to tumor necrosis factor-related apoptosisinducing ligand. Mol Cancer Res. 2007;5:615-25.

40. Greenblatt MS, Bennett WP, Hollstein M, Harris CC. Mutations in the p53 tumor suppressor gene: clues to cancer etiology and molecular pathogenesis. Cancer Res. 1994;54:4855-78.

41. Levine AJ. p53, the cellular gatekeeper for growth and division. Cell. 1997;88:323-31.

42. Takimoto R, El-Deiry WS. Wild-type p53 transactivates the KILLER/DR5 gene through an intronic sequence-specific DNAbinding site. Oncogene. 2000;19:1735-43.

43. Takeda K, Stagg J, Yagita H, Okumura K, Smyth MJ. Targeting death-inducing receptors in cancer therapy. Oncogene. 2007;26: $3745-57$.

44. Grosse-Wilde A, Voloshanenko O, Bailey SL, Longton GM, Schaefer U, Csernok AI, et al. TRAIL-R deficiency in mice enhances lymph node metastasis without affecting primary tumor development. J Clin Invest. 2008;118:100-10.

45. Finnberg N, Klein-Szanto AJ, El-Deiry WS. TRAIL-R deficiency in mice promotes susceptibility to chronic inflammation and tumorigenesis. J Clin Invest. 2008;118:111-23.

46. Tecchio C, Huber V, Scapini P, Calzetti F, Margotto D, Todeschini $\mathrm{G}$, et al. IFN $\alpha$-stimulated neutrophils and monocytes release a soluble form of TNF-related apoptosis-inducing ligand (TRAIL/Apo-2 ligand) displaying apoptotic activity on leukemic cells. Blood. 2004;103:3837-44.

47. Altucci L, Rossin A, Raffelsberger W, Reitmair A, Chomienne C, Gronemeyer H. Retinoic acid-induced apoptosis in leukemia cells is mediated by paracrine action of tumor-selective death ligand TRAIL. Nat Med. 200;7:680-6.

48. Clarke N, Jimenez-Lara AM, Voltz E, Gronemeyer H. Tumor suppressor IRF-1 mediates retinoid and interferon anticancer signaling to death ligand TRAIL. EMBO J. 2004;23:3051-60.

49. Ludwig AT, Moore JM, Luo Y, Chen X, Saltsgaver NA, O'Donnell MA, et al. Tumor necrosis factor-related apoptosisinducing ligand: a novel mechanism for Bacillus Calmette-Guerin-induced antitumor activity. Cancer Res. 2004;64:3386-90. 